\title{
The effect of copper vacancies on the anion position of chalcopyrite type $\mathrm{CuGaS}_{2}$
}

\author{
Julien Marquardt ${ }^{1,2}$, Galina Gurieva ${ }^{1}$, Christiane Stephan-Scherb ${ }^{2,3}$, Susan Schorr ${ }^{1,2}$
}

\begin{abstract}
${ }_{1}$ Helmholtz-Zentrum für Materialien und Energie, Hahn-Meitner-Platz 1, 14109 Berlin, Germany
2 Freie Universität Berlin, Institut für Geologische Wissenschaften, Malteserstr. 74-100, 12249 Berlin, Germany

3 Bundesanstalt für Materialforschung und -prüfung, Unter den Eichen 87, 12205 Berlin, Germany
\end{abstract}

The prediction of structural parameters and optoelectronic properties of compound semiconductors is very important. However, calculations often neglect chemical variability and structural defects. In chalcopyrite type semiconductors one of the major defects are copper vacancies $\left(\mathrm{V}_{\mathrm{Cu}}\right)$. The four cation neighbors of the anion determine its position in the chalcopyrite type structure expressed by the Wyckoff position $8 \mathrm{~d}(x, 1 / 4,1 / 8)$. Intrinsic point defects like $\mathrm{V}_{\mathrm{Cu}}$ and anti-sites may cause variations of the anion position in the middle of the cation tetrahedron, especially in the anion position parameter $x$. For stoichiometric chalcopyrite type compounds a formalism according to the principle of conservation of tetrahedral bonds (CTB) can be applied to calculate the anion position parameter, but it fails in the case of offstoichiometric chalcopyrites. This case study of chalcopyrite type $\mathrm{CuGaS}_{2}$ and $\mathrm{Mn}$-substituted $\mathrm{GuGaS}_{2}$ shows that the experimentally determined anion position parameter $x$ deviate from values calculated by CTB approach. The systematic investigation of off-stoichiometric $\mathrm{CuGaS}_{2}$ and $\mathrm{Mn}$-substituted $\mathrm{GuGaS}_{2}$ demonstrates the effect of copper vacancies on the average radii of the cation sites (Wyckoff positions $4 \mathrm{a}$ and $4 \mathrm{~b}$ ) as well as on the anion position parameter $x$. By applying an elaborated CTB approach implementing copper vacancies an agreement between experimental and calculated anion position parameter $x$ could be obtained. 


\section{Introduction}

The ternary chalcopyrite type compound $\mathrm{CuGaS}_{2}$ shows a certain chemical variability within the stability region [1,2]. These stoichiometry deviations result in several intrinsic point defects (vacancies, anti-sites and interstitials) which do lead to changes of structural parameters. First, the chalcopyrite type lattice parameters are affected and most important, due to the changes in chemical composition, the cation distribution is influenced. Furthermore, the cation distribution (varied by the presence of anti-sites) impacts the position of the anion within the chalcopyrite type structure, which is in turn affecting the band gap of this type of semiconductor material. In chalcopyrite type $\mathrm{CuGaS}_{2}$ (space group $I \overline{4} 2 d$ ), copper occupies the Wyckoff position $4 \mathrm{a}(0$, 0,0), gallium occupies the Wyckoff position $4 \mathrm{~b}(0,0,0.5)$ and sulfur occupies the Wyckoff position $8 \mathrm{~d}(x, 0.25,0.125)$ [3]. This means, the anion position can shift along the crystallographic $\vec{a}$ direction. As a first order approximation, the $x$ coordinate of the anion, also called anion parameter $x$, depends on the ratio of the cations at the $4 \mathrm{a}$ and $4 \mathrm{~b}$ site. The normalized anion parameter $x$, named tetragonal distortion $u$, represents the absolute deviation from the ideal anion position in the middle of the cation tetrahedron $(x=0.25)$. The displacement of the anion results in different cation-anion bond length $\mathrm{R}_{\mathrm{AC}}$ and $\mathrm{R}_{\mathrm{BC}}$ (according to the general formula of $\mathrm{ABX}_{2}$ chalcopyrite type compounds), which in turn cause different-sized anion tetrahedra $\left(\mathrm{AC}_{4}\right.$ and $\left.\mathrm{BC}_{4}\right)$, resulting in a tetragonal deformation, $\eta=\mathrm{c} / 2 \mathrm{a}$, in the direction of the crystallographic $\vec{c}$ axis. Jaffe and Zunger developed the principle of the conservation of tetrahedral bonds (CTB) for ternary $\mathrm{ABC}_{2}$ chalcopyrites [4], in which the bond mismatch parameter is defined as

$$
\alpha=R_{A C}^{2}-R_{B C}^{2}=\left(r_{A}+r_{C}\right)^{2}-\left(r_{B}+r_{c}\right)^{2}
$$

and the mean-square bond as

$$
\beta=R_{A C}^{2}+R_{B C}^{2}=\left(r_{A}+r_{C}\right)^{2}+\left(r_{B}+r_{C}\right)^{2}
$$

where $r_{A}, r_{B}$ and $r_{C}$ are the cation and anion radii, respectively. 
According to the CTB principle the lattice parameter $a$ and the tetragonal deformation $\eta$ can be calculated using the bond mismatch parameter $\alpha$ and the mean-square bond $\beta$ [4]

$$
\begin{aligned}
& a^{2}=\frac{12 \alpha^{2}}{2 \beta+\alpha-\left[(2 \beta+\alpha)^{2}-18 \alpha^{2}\right]^{1 / 2}}, \\
& \eta^{2}=\frac{8(\beta-\alpha)}{3 a^{2}} .
\end{aligned}
$$

Applying the Abraham and Bernstein equation [5], which fixes the tetrahedral bond constraint around the B cation given the anion parameter $x$ can be calculated

$$
x=\frac{1}{2}-\frac{1}{4}\left(2 \eta^{2}-1\right)^{1 / 2} .
$$

The anion parameter $x$ is normalized by

$$
u=|0.25-x|
$$

which results in the tetragonal distortion $u$ [4].

The formalism (equations 1-4) developed by Jaffe and Zunger [1] can be used to calculate the tetragonal distortion $u$ for a chalcopyrite type compound using only the radii of the involved c. It was successfully applied in the study of stoichiometric Chalcopyrite type $\mathrm{Zn}_{2 \mathrm{x}}(\mathrm{CuB})_{1-\mathrm{x}} \mathrm{C}_{2}$ mixed crystals [6], where the effect of the cation distribution was noticed by modifying eq. (1) and (2) using an average radius of the Wyckhoff sites $4 \mathrm{a}$ and $4 \mathrm{~b}$ taking into account anti-site defects.

This work focuses on structural changes of the chalcopyrite type Mn substituted $\mathrm{CuGaS}_{2}$. Experimentally obtained $x$ values in $\mathrm{CuGaS}_{2}$ show a wide spread from $x=0.25$ to $x=0.275$ [5-8] due to the chemical flexibility (off-stoichiometry) of the material. It was shown, that $\mathrm{CuGaS}_{2}$ accepts deviations from stoichiometry by keeping the chalcopyrite type crystal structure [9]. Nevertheless the chemical variations in off-stoichiometric $\mathrm{CuGaS}_{2}$ induce only small structural changes. The incorporation of another cation, like Mn, enhances such changes, as it was demonstrated by incorporating $\mathrm{Zn}$ in other chalcopyrites [10].

The systematic study presented here was performed on a series of Mn-substituted $\mathrm{CuGaS}_{2}$ powder samples. The lattice parameter and the tetragonal distortion were determined by 
Rietveld analysis of X-ray diffraction data. The cation distribution on the two structural sites $4 \mathrm{a}$ and $4 \mathrm{~b}$ was determined by the average neutron scattering length analysis method of neutron diffraction data allowing the determination of cation anti-site defects as well as cation vacancies [11]. Due to the negative coherent neutron scattering length of manganese and the difference in the coherent neutron scattering length of copper and gallium, these electronic similar elements can be distinguished in the Rietveld analysis of neutron diffraction data. In this work an advanced CTB principle formalism was developed to take the effect of copper vacancies $\left(\mathrm{V}_{\mathrm{Cu}}\right)$ into account. An agreement between the experimentally determined tetragonal distortion $u$ and values calculated by the advanced formalism could be obtained.

\section{Experimental}

Powder samples were synthesized by solid state reaction of pure elements in closed silica tubes, following the pseudo-binary sections of $\mathrm{Cu}_{0.5}\left(\mathrm{Ga}_{0.5-\mathrm{x}} \mathrm{Mn}_{\mathrm{x}}\right) \mathrm{S}$ and $\left(\mathrm{Cu}_{0.5} \mathrm{Ga}_{0.5} \mathrm{~S}\right)_{1-\mathrm{x}}(\mathrm{MnS})_{\mathrm{x}}$. The elements were weighed-in referring their specific chemical composition calculated according to both of the series above, adding $10 \%$ sulfur excess to avoid a sulfur deficit, and placed in a carbon boat. The synthesis was performed in a one-zone furnace at temperatures of $800-950^{\circ} \mathrm{C}$ for 300 h. Later on two homogenization steps followed (grinding in an agate mortar, pressing pellets, heat treatment at $900-950^{\circ} \mathrm{C}$ for $300 \mathrm{~h}$ in closed silica tubes). The synthesis and annealing temperatures were varied to improve the solid state reaction.

The chemical composition of the chalcopyrite type phase as well as of secondary phases was determined by wavelength dispersive X-ray spectroscopy (WDX) using an electron microprobe analysis unit (JEOL-JXA 8200) equipped with 5 spectrometers. To ensure an appropriate measurement, the system was calibrated by elemental standards. For each powder sample 50 grains (10 measurement points each) of the chalcopyrite type phases were analyzed and averaged. For the secondary phases, the number of measured grains was reduced. 
X-ray powder diffraction data were collected using a Panalytical MPD operating with $\mathrm{Cu} \mathrm{K} \alpha$ radiation in Bragg-Brentano geometry. To address the instrumental effects on the XRD pattern an instrumental resolution function based on a refinement of lanthanum hexa-boride $\left(\mathrm{LaB}_{6}\right)$ was used. Thereafter a full pattern Rietveld refinement using the FullProf Suite 3.0 [12] was performed. As starting model for the structural refinement, the chalcopyrite type structure as well as the corresponding structures of the secondary phases were applied. The sequence of the refinement steps can be found elsewhere [13]. An overview of the chemical composition of the chalcopyrite type phases, the obtained tetragonal distortion $u$ as well as the average radii of the cation sites $4 \mathrm{a}$ and $4 \mathrm{~b}$ can be found in Table 1 . The latter were obtained by subtracting the crystal radius of sulfur $\left(r_{S^{2-}=1.7 \AA} \AA\right.$ [14]) from the bond lengths (4a)-S and (4b)-S experimentally determined by the Rietveld analysis of XRD data. The copper vacancy fraction is calculated from the chemical composition obtained by WDX spectroscopy.

Neutron powder diffraction experiments were performed using the Fine Resolution Powder Diffractometer (FIREPOD, E9) at the Berlin Research Reactor (BERII) with a wavelength of 1.7982(1) A [15]. Each neutron diffraction (ND) pattern was treated by a Rietveld refinement comparable to the analysis of the XRD data. The cation distribution was determined from the obtained cation site occupancy factors (SOF) by applying the average neutron scattering length analysis $[9,11,13]$. Details on the determination of the cation distribution can be found elsewhere [16].

\section{Results and discussion}

The experimentally determined tetragonal distortion $u$ for the studied $\mathrm{CuGaS}_{2}$ is $u=0.0112(13)$. However, the value of the tetragonal distortion calculated from the crystal radii [14] according to the CTB principle for stoichiometric $\mathrm{CuGaS}_{2}$ is $u=0.0088$. In literature values from $u=0$ to $u=0.025$ [5-8] were reported. The reason for such a spread in the reported values might be due 
to off-stoichiometric composition resulting in different concentrations of structural defects, such as copper vacancies.

Besides the tetragonal distortion $u$ of $\mathrm{CuGaS}_{2}$ the corresponding values for the two hypothetical endmembers $\mathrm{CuMnS}_{2}$ and $\mathrm{MnGaS}_{2}$ were calculated according to the CTB principle using the crystal radii from Shannon [14] (see Table 2). These three values may serve as initial points to apply Vegard's law [15] to visualize trends in the variation of structural parameters due to a manganese incorporation into the chalcopyrite type structure (see Figure 1, red lines). If manganese $\left(\mathrm{r}_{\mathrm{Mn}}{ }^{2+}=0.725 \AA[14]\right)$ substitutes copper $\left(\mathrm{r}_{\mathrm{Cu}}{ }^{+}=0.635 \AA[14]\right)$ and occupies the $4 \mathrm{a}$ site, the average radius of the $4 \mathrm{a}$ site increases which results in an increase in tetragonal distortion $u$. The opposite trend is observed for manganese substituting gallium $\left(\mathrm{r}_{\mathrm{Ga}}{ }^{3+}=0.580 \AA\right.$ [14]), in this case the tetragonal distortion $u$ decreases to zero, were both cation sites are equal in average size and increases afterwards with increasing manganese content. This behavior results from the absolute character of the tetragonal distortion $u(u \geq 0)$, the anion parameter $x$ would only decrease. These trends of the tetragonal distortion $u$ are compared with experimentally determined $u$ values from Rietveld refinement of XRD data (see Figure 1, red lines). The experimentally determined tetragonal distortion follows a similar trend but with a certain shift from the prediction. Tetragonal distortion values which fall below the predicted trend may be influenced by $\mathrm{Cu}_{\mathrm{Ga}}$ and $\mathrm{Ga}_{\mathrm{Cu}}$ anti-site defects. Thus, the average radii of the $4 \mathrm{a}$ and $4 \mathrm{~b}$ site become more equal in size. However, tetragonal distortion values plotting above the predicted trend cannot be explained by $\mathrm{Cu}_{\mathrm{Ga}}$ and $\mathrm{GaCu}$ anti-site defects. To make this effect visible, the data points in Figure 1 were color coded according to the concentration of $\mathrm{Cu}$ vacancies. Additionally the tetragonal distortion $u$ of $\mathrm{CuGaS}_{2}$ and the two hypothetical endmembers $\mathrm{CuMnS}_{2}$ and $\mathrm{MnGaS}_{2}$ were calculated according to the CTB principle again but assuming $7.5 \%$ vacancies on the $4 \mathrm{a}$ site. If now these three values serve as initial points to apply Vegard's law [15] to visualize trends in the variation of structural parameters a clear upshift of the trendlines are observed (see Figure 1, blue lines). 
To include copper vacancies properly in the CTB principle formalism, first the effect on the crystal structure has to be evaluated. Considering the chalcopyrite type structure, the presence of a copper vacancy would result in four degenerated tetrahedra, containing one copper atom and two gallium atoms each (Figure 2). The sulfur atoms are dragged into the direction of the remaining atom and apart from each other. Resulting from this, one copper vacancy influences four $\mathrm{Cu}-\mathrm{S}$ and eight $\mathrm{Ga}-\mathrm{S}$ bond length. However, to include this in the calculation, three assumptions need to be taken into account. The first assumption has the physical meaning to maintain the symmetry of the tetrahedra, in order to keep the space group symmetry, and ultimately the chalcopyrite type structure. Therefore the affected $\mathrm{Cu}-\mathrm{S}$ and $\mathrm{V}_{\mathrm{Cu}}-\mathrm{S}$ bond lengths are assumed to be equal in the corresponding tetrahedra. The second assumption is that manganese does not occupy tetrahedra which are affected by a copper vacancy. Also, anti-site defects are not considered in the degenerated tetrahedra. The second and the third assumption are made to simplify the calculation and to minimize the number of variables. In this way only the formalism of calculating the average radii of the $4 \mathrm{a}$ and $4 \mathrm{~b}$ site of the chalcopyrite type structure is changed. The calculation of the bond mismatch parameter $\alpha$, mean-square bond $\beta$, $a^{2}$ and $\eta^{2}$ remain according to the CTB principle as introduced by Jaffe and Zunger [4] (equ. 1 -4) as well as the calculation of the anion parameter $x$ defined by Abrahams and Bernstein [5] (equ. 5). Taking into account copper vacancies, the average radii of the $4 \mathrm{a}$ and $4 \mathrm{~b}$ sites are now calculated by

$$
\begin{aligned}
& r_{A}=r_{C u} *\left(x_{C u}-4 x_{V_{C u}}\right)+r_{C u L} * 4 x_{V_{C u}}+r_{V_{C u}} * x_{V_{C u}}+r_{G a} * x_{G a}+r_{M n} * x_{M n}+r_{S} \\
& \rightarrow r_{A}=r_{C u} *\left(x_{C u}-4 x_{V_{C u}}\right)+r_{C u L} * 5 x_{V_{C u}}+r_{G a} * x_{G a}+r_{M n} * x_{M n}+r_{S} \\
& r_{B}=r_{G a} *\left(x_{G a}-8 x_{V_{C u}}\right)+r_{G a S} * 8 x_{V_{C u}}+r_{C u} * x_{C u}+r_{M n} * x_{M n}+r_{S}
\end{aligned}
$$


were $r_{C u L}$ represents an increased copper radius and $r_{G a S}$ a decreased gallium radius (relative to the crystal radii of $\mathrm{Cu}^{+}$and $\mathrm{Ga}^{3+}$ ), and $x$ describes the fraction of the subscribed element or vacancy on this site. Based on systematic variations of the increased copper radius $\left(r_{C u L}\right)$ and the reduced gallium radius $\left(r_{G a S}\right)$ the minimum increase and decrease of the average radii of the two cationic sites necessary to represent the experimentally obtained tetragonal distortion $u$ was determined to be 5\%. The according changes of the bond lengths are even smaller, less than 1.4 $\%$. The $\mathrm{Cu}-\mathrm{S}$ bond length in tetrahedra which are affected by a copper vacancy increase from $2.335 \AA$ to $2.367 \AA$, whereas the Ga-S bond length decreases from $2.280 \AA$ to $2.251 \AA$.

The advanced formalism was applied to calculate the tetragonal distortion $u$ for Mn-substituted $\mathrm{CuGaS}_{2}$ taking into account the cationic point defects experimentally determined by the average neutron scattering length analysis of neutron diffraction data [16]. It has to be noted that this analysis was performed only with those samples which contain no or only a small amount of secondary phases (see table 1 , marked in bold). As a first step the average radii of the cation sites $4 \mathrm{a}$ and $4 \mathrm{~b}$ were calculated according to equations (7) and (8) taking into account copper vacancies and anti-site defects determined in the neutron diffraction study [16].

A good agreement between experimental and calculated average radii values can be obtained for chalcopyrite-type phases with $\mathrm{Cu} /(\mathrm{Ga}+\mathrm{Mn})>0.78$ (see Figure 3). In a second step the tetragonal distortion $u$ was calculated according to CTB principle formalism (equ. 1-4) and the Abraham-Bernstein equation (equ. 5) using the average radii of the cation sites $4 \mathrm{a}$ and $4 \mathrm{~b}$ (as explained above). Again for the chalcopyrite-type phases with $\mathrm{Cu} /(\mathrm{Ga}+\mathrm{Mn})>0.78$ a good agreement between experimental and calculated values can be obtained (figure 4). This also shows the limit of the method: if the cation ratio value falls below a certain limit, that means the chalcopyrite-type phase becomes very copper poor, the advanced formalism is no longer valid. Thus the advanced CTB principle formalism enhances the predictability of structural changes due to copper vacancies up to a certain limit beyond which the actual anion displacement is more complex. If the chalcopyrite-type phase becomes very copper poor the 
anion within the degenerated tetrahedra will be pulled into the direction of the three remaining cations, but mainly in the direction of gallium. The resulting anion displacement is comparable to those of the stannite or kesterite type structure (space group $I \overline{4} 2 m$ and $I \overline{4}$, respectively) with the anion coordinated by two cation of the same size and two larger which differ in size. Here the anion shifts in two or all three directions, resulting in a more general anion position with the atomic coordinates $(\mathrm{x}, \mathrm{x}, \mathrm{z})$ and $(\mathrm{x}, \mathrm{y}, \mathrm{z})$ respectively [17]. This anion displacement causes a break in symmetry. By a combination of neutron diffraction and anomalous X-ray scattering it was shown that the vacancy compound $\mathrm{CuGa}_{3} \mathrm{Se}_{5}$, which is formed as a secondary phase besides $\mathrm{Cu}$-poor chalcopyrite-type $\mathrm{CuGaSe}_{2}$ if $\mathrm{Cu} / \mathrm{Ga}<0.78$ [18], adopts a modified stannite type structure [19].

However, the advanced formalism presented here is a the first step (taking into account copper vacancies), towards a general understanding of the influence of off-stoichiometry on the anion displacement in $\mathrm{CuGaS}_{2}$ and other chalcopyrite-type phases for which a complex scenario of intrinsic point defects have to be taken into account especially for compositions far from stoichiometry.

\section{Conclusion}

The effect of copper vacancies on the average radii of the cation sites $4 \mathrm{a}$ and $4 \mathrm{~b}$ as well as on the tetragonal distortion $u$ could be shown. Furthermore, it was possible to enhance the CTB principle formalism [1] with regard of the influence of copper vacancies and anti-site defects. A copper vacancy causes four degenerated coordination tetrahedra, were the anion is dragged into the direction of the gallium atoms. Resulting from this the Ga-S bond length decreases, whereas the $\mathrm{Cu}-\mathrm{S}$ bond length increases. The enhanced CTB principle formalism was verified by applying it to the off-stoichiometric chalcopyrite-type phase in the $\mathrm{Cu}\left(\mathrm{Ga}_{1-\mathrm{x}} \mathrm{Mn}_{\mathrm{x}}\right) \mathrm{S}_{2}$ and 
$\left(\mathrm{Cu}_{0.5} \mathrm{Ga}_{0.5} \mathrm{~S}\right)_{1-\mathrm{x}}(\mathrm{MnS})_{\mathrm{x}}$ series. The cation distribution used in the advanced formalism was determined by the averaged neutron scattering length analysis of neutron diffraction data.

It could be shown that the advanced CTB principle formalism developed here revealed a considerably better agreement between calculated and experimental values for the average radii of the cation sites $4 \mathrm{a}$ and $4 \mathrm{~b}$ site as well as for the tetragonal distortion $u$ in Mn-substituted chalcopyrite-type $\mathrm{CuGaS}_{2}$.

\section{Acknowledgement}

This research was financially supported by the HZB Graduate School "Materials for Solar Energy Conversion" (MatSEC). Special thanks to Frederike Lehmann and René Gunder from HZB for support and fruitful discussions. 
[1] M. Kokta, J. R. Carruthers, M. Grasso, H. M. Kasper, and B. Tell, J. .Electron. Mater. 1976, 5, 69-89.

[2] S. Stephan, Schriftenreihe des HZB 2011, B17, doi: httpx://dx.doi.org/10.5442/d0011

[3] S. Hall, Can. Mineral. 1975, 13, 168-172.

[4] J. E. Jaffe and A. Zunger, Phys. Rev. B 1984, 29, 1882-1906.

[5] S. Abrahams and J. Bernstein, J. Chem. Phys. 1973, 59, 5415-5422.

[6] S. Schorr, C. Stephan, C. A. Kaufmann, in: Neutron Applications in Materials for Energy, 2015, ed. by G. J. Kearly and V. K. Peterson, Springer.

[7] H. Spiess, U. Haeberlen, G. Brandt, A. Räuber, and J. Schneider, Phys. Status Solidi B 1974, 62, 183-192.

[8] J. Schneider, A. Räuber, and G. Brandt, J. Phys. Chem. Solids 1973, 34, 443-450.

[9] C. Stephan, T. Scherb, C. A. Kaufmann, S. Schorr, and H. W. Schock, Appl. Phys. Lett. 2012, 101, 101907.

[10] S. Schorr, M. Tovar, D. Sheptyakov, L. Keller, G. Geandier, J. Phys. Chem. Sol. 2005, 66, 19611965.

[11] S. Schorr, Ch. Stephan, D. Többens, R. Gunder, T. Törndal, in: Advanced characterization techniques for thin film solar cells 2016, ed. by D. Abou-Ras, T. Kirchartz, U. Rau, Wiley

[12] J. Rodriguez-Carvajal, "FULLPROF version 3.0. 0," Laboratorie Leon Brillouin, CEA-CNRS, 2003.

[13] C. Stephan, S. Schorr, M. Tovar, and H.-W. Schock, Appl. Phys. Lett. 2011, 98, 091906.

[14] R. D. Shannon, in: Structure and Bonding in Crystals 1981, ed. M. O'Keeffe, A. Navrotsky, Vol. II, Academic Press, New York, 53-70.

[16] J. Marquardt, S. Levcenko, A. Franz, T. Unold, C. Stephan-Scherb, and S. Schorr, Journal of Alloys and Compounds 2019, submitted.

[17] S. R. Hall, J. T. Szymanski, J. M. Stewart, Can. Mineral. 1978, 16, 131 - 137.

[18] Ch. Stephan, S. Schorr, H.-W. Schock, Mater. Res. Soc. Symp. Proc. 2009, 1165 1165-M0908 .

[19] S. Lehmann, D. Fuertes Marrón, M. León, R. Feyerherm, E. Dudzik, E. J. Friedrich, M. Tovar, Y. Tomm, C. Wolf, S. Schorr, Th. Schedel-Niedrig, M. Ch. Lux-Steiner and J. M. Merino, J. Appl. Phys. 2011, 109, 013518. 
Table 1 Overview of chemical and structural parameters of chalcopyrite type Mn-substituted $\mathrm{CuGaS}_{2}$. Chemical composition is expressed by the cation ratios $\mathrm{Cu} / \mathrm{Ga}$ and $\mathrm{Cu} /(\mathrm{Ga}+\mathrm{Mn})$ as well as the metal to sulfur ratio $(\mathrm{M} / \mathrm{S})$. $\mathrm{x}_{\mathrm{m}}$ gives the mole faction of manganese in $\left[\mathrm{Cu}_{0.5} \mathrm{Ga}_{0.5} \mathrm{~S}\right]_{1-}$ ${ }_{x}-[M n S]_{x}$. Further listed are the relative amount of copper vacancies $\left(\mathrm{V}_{\mathrm{Cu}}\right)$, the radii of the cation sites $4 \mathrm{a}$ and $4 \mathrm{~b}$ as well as the tetragonal distortion $\mathrm{u}$. Samples, for which the advanced model was applied are in bold.

\begin{tabular}{|c|c|c|c|c|c|c|c|c|}
\hline & $\mathrm{x}_{\mathrm{m}}$ & $\mathrm{Cu} / \mathrm{Ga}$ & $\mathrm{Cu} /(\mathrm{Ga}+\mathrm{Mn})$ & $\mathrm{M} / \mathrm{S}$ & $\mathrm{V}_{\mathrm{Cu}}[\%]$ & $4 a$ & $4 b$ & $\mathrm{u}$ \\
\hline & 0 & $1.00(2)$ & $1.00(2)$ & $0.97(2)$ & $2.5(5)$ & $0.638(4)$ & $0.569(4)$ & $0.0112(13)$ \\
\hline \multirow{8}{*}{ 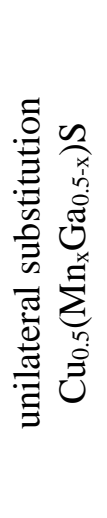 } & $0.012(1)$ & $0.99(2)$ & $0.97(2)$ & $0.99(2)$ & $2.2(5)$ & $0.641(3)$ & $0.571(3)$ & $0.0112(8)$ \\
\hline & $0.063(1)$ & $0.93(2)$ & $0.82(2)$ & $0.98(2)$ & $3.6(5)$ & $0.644(6)$ & $0.578(5)$ & $0.0106(18)$ \\
\hline & $0.079(2)$ & $0.93(2)$ & $0.79(2)$ & $0.98(2)$ & $3.6(5)$ & $0.638(4)$ & $0.587(4)$ & $0.0081(14)$ \\
\hline & $0.085(2)$ & $0.92(2)$ & $0.78(2)$ & $0.99(2)$ & $2.9(5)$ & $0.660(4)$ & $0.568(4)$ & $0.0147(12)$ \\
\hline & $0.040(1)$ & $0.97(2)$ & $0.90(2)$ & $0.99(2)$ & $1.3(5)$ & $0.641(4)$ & $0.572(4)$ & $0.0111(11)$ \\
\hline & $0.061(1)$ & $1.03(2)$ & $0.91(2)$ & $1.00(2)$ & $0.9(5)$ & $0.622(6)$ & $0.582(6)$ & $0.0012(20)$ \\
\hline & $0.071(1)$ & $1.00(2)$ & $0.87(2)$ & $0.99(2)$ & $1.4(5)$ & $0.627(6)$ & $0.596(6)$ & $0.0052(24)$ \\
\hline & $0.081(2)$ & $0.97(2)$ & $0.83(2)$ & $1.01(2)$ & $0(5)$ & $0.656(5)$ & $0.596(5)$ & $0.0024(14)$ \\
\hline \multirow{12}{*}{ 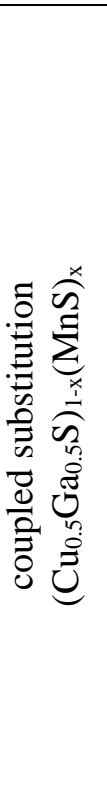 } & $0.021(1)$ & $1.00(2)$ & $0.96(2)$ & $0.97(2)$ & $5.5(5)$ & $0.639(4)$ & $0.573(4)$ & $0.0105(12)$ \\
\hline & $0.041(1)$ & $1.00(2)$ & $0.92(2)$ & $0.97(2)$ & $5.3(5)$ & $0.642(4)$ & $0.574(4)$ & $0.0109(13)$ \\
\hline & $0.082(2)$ & $0.91(2)$ & $0.77(2)$ & $0.97(2)$ & $6.8(5)$ & $0.659(3)$ & $0.559(3)$ & $0.0160(10)$ \\
\hline & $0.091(2)$ & $0.97(2)$ & $0.80(2)$ & $0.97(2)$ & $6.1(5)$ & $0.643(4)$ & $0.574(4)$ & $0.0111(12)$ \\
\hline & $0.014(1)$ & $0.95(2)$ & $0.93(2)$ & $0.97(2)$ & $6.7(5)$ & $0.644(3)$ & $0.564(2)$ & $0.0128(8)$ \\
\hline & $0.041(1)$ & $0.93(2)$ & $0.85(2)$ & $0.96(2)$ & $7.0(5)$ & $0.650(3)$ & $0.564(2)$ & $0.0137(8)$ \\
\hline & $0.063(1)$ & $0.92(2)$ & $0.81(2)$ & $0.96(2)$ & $7.1(5)$ & $0.654(3)$ & $0.562(2)$ & $0.0146(8)$ \\
\hline & $0.069(1)$ & $1.02(2)$ & $0.89(2)$ & $0.99(2)$ & $1.9(5)$ & $0.619(9)$ & $0.600(9)$ & $0.0028(28)$ \\
\hline & $0.063(1)$ & $0.95(2)$ & $0.84(2)$ & $0.98(2)$ & $4.3(5)$ & $0.651(5)$ & $0.568(4)$ & $0.0134(14)$ \\
\hline & $0.087(2)$ & $1.00(2)$ & $0.84(2)$ & $0.99(2)$ & $1.9(5)$ & $0.622(16)$ & $0.604(15)$ & $0.0026(47)$ \\
\hline & $0.089(2)$ & $1.00(2)$ & $0.83(2)$ & $0.99(2)$ & $2.8(5)$ & $0.629(16)$ & $0.598(15)$ & $0.0051(48)$ \\
\hline & $0.098(2)$ & $0.91(2)$ & $0.75(2)$ & $0.96(2)$ & $7.1(5)$ & $0.639(6)$ & $0.577(6)$ & $0.0104(20)$ \\
\hline
\end{tabular}

Table 2 Tetragonal distortion $u$ of the endmembers calculated by the CTB principle (equ. 1-4) and the Abrahams-Bernstein equation (equ. 5), (-) is marking negative values.

endmember $\quad u$

$\mathrm{CuGaS}_{2} \quad 0.0088$

$\mathrm{MnGaS}_{2} \quad 0.0225$

$\mathrm{CuMnS}_{2} \quad 0.0144(-)$ 

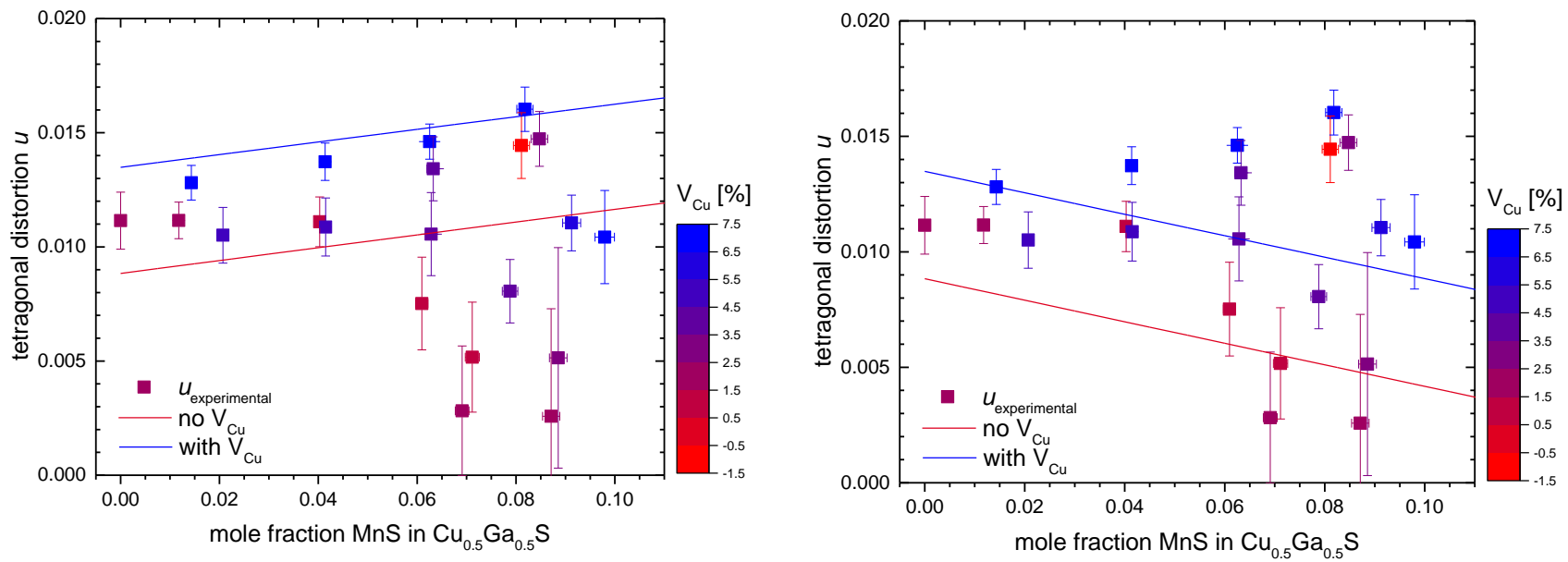

Figure 1 Experimentally obtained tetragonal distortion $u$ in dependence on the manganese contend in chalcopyrite-type Mn-substituted $\mathrm{CuGaS}_{2}$.

The data points are color-coded referring by corresponding to the copper vacancy fraction calculated from chemical composition (Table 1). The figures present two limiting cases concerning an assumed manganese cation site occupation: left - the substituted manganese occupies the $4 \mathrm{a}$ site exclusively, right - the substituted manganese occupies the $4 \mathrm{~b}$ site exclusively.

The lines presents trends of the tetragonal distortion evaluated by Vegard's law using $u$ values calculated by the CTB principle for $\mathrm{CuGaS}_{2}$ and the two endmembers $\mathrm{CuMnS}_{2}$ and $\mathrm{MnGaS}_{2}$ (see table 2). The presence of copper vacancies was taken into account (red line - no $\mathrm{V}_{\mathrm{Cu}}$, blue line - assuming $7.5 \% \mathrm{~V}_{\mathrm{Cu}}$ ).
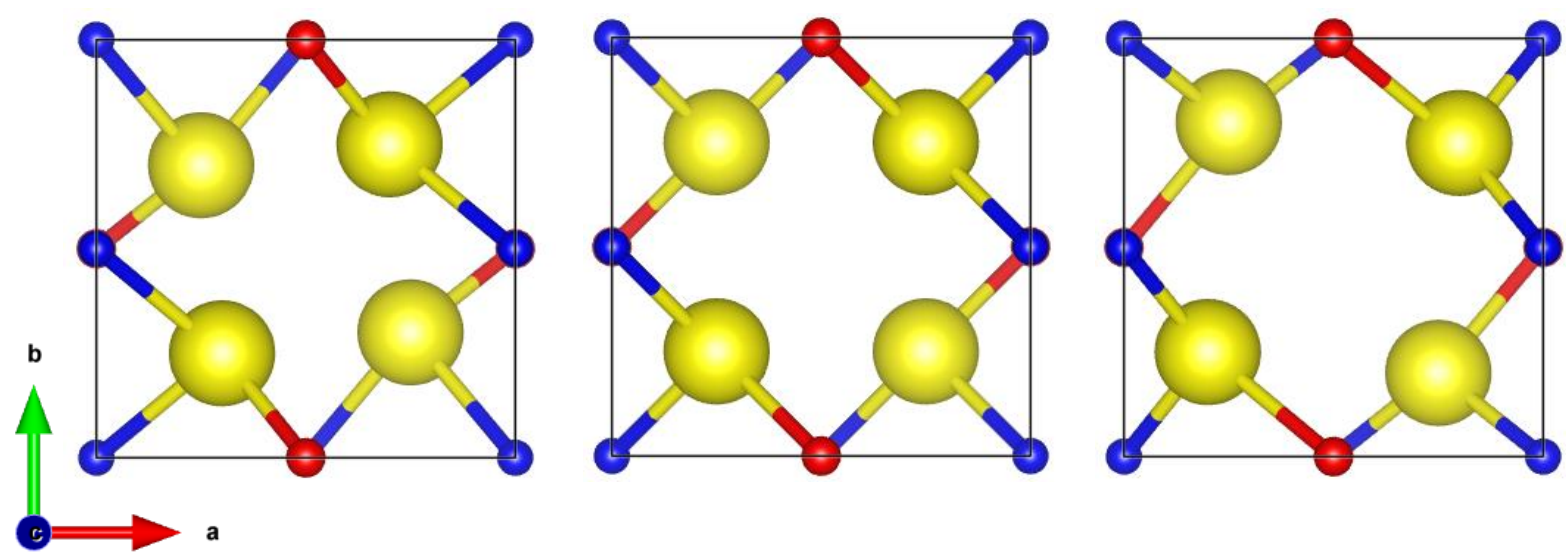

Figure 2 Presentation of a cut within the $\vec{a}-\vec{b}$ plane in the chalcopyrite type structure around a copper vacancy with an anion position of $\mathrm{x}=0.2$ (left), $\mathrm{x}=0.25$ (middle) and $\mathrm{x}=0.3$ (right) $(\mathrm{Cu}-$ red, $\mathrm{Ga}$ - blue and $\mathrm{S}$-yellow). 

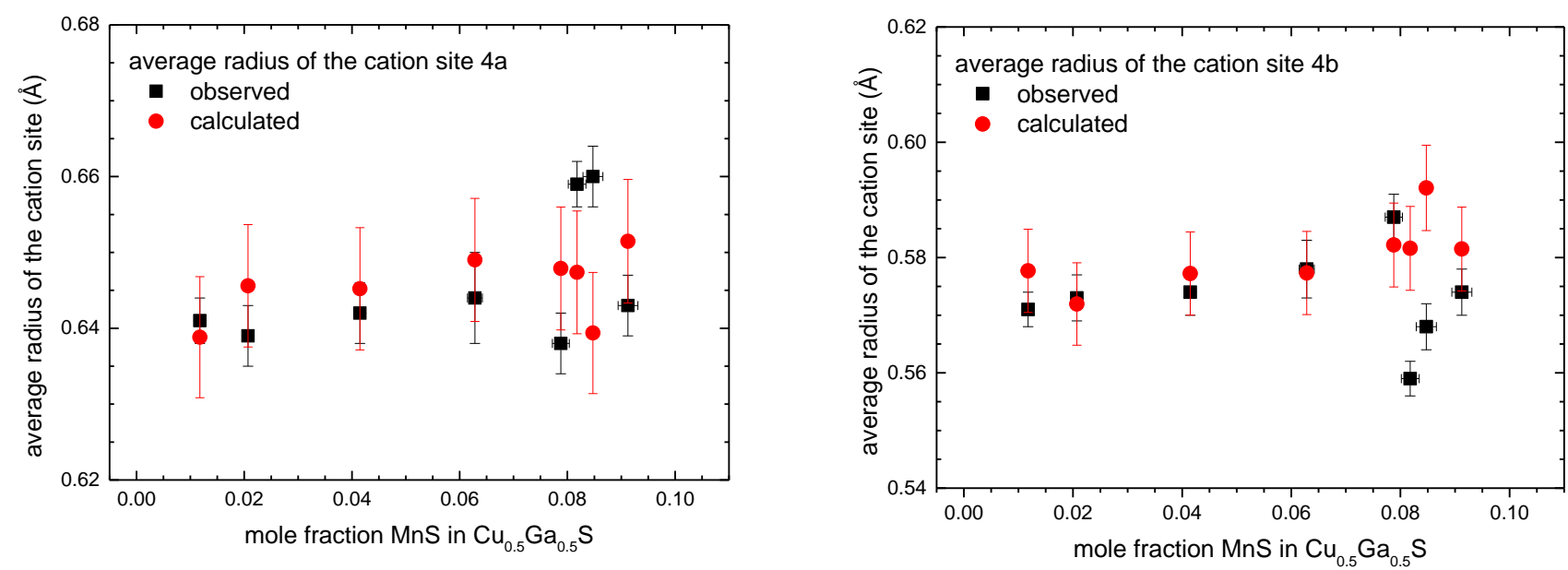

Figure 3 Comparison of experimentally observed and calculated average radii of the cation sites $4 \mathrm{a}$ (left) and 4b (right) in chalcopyrite-type Mn-substituted $\mathrm{CuGaS}_{2}$. For the calculation the advanced CTB principle formalism was applied.

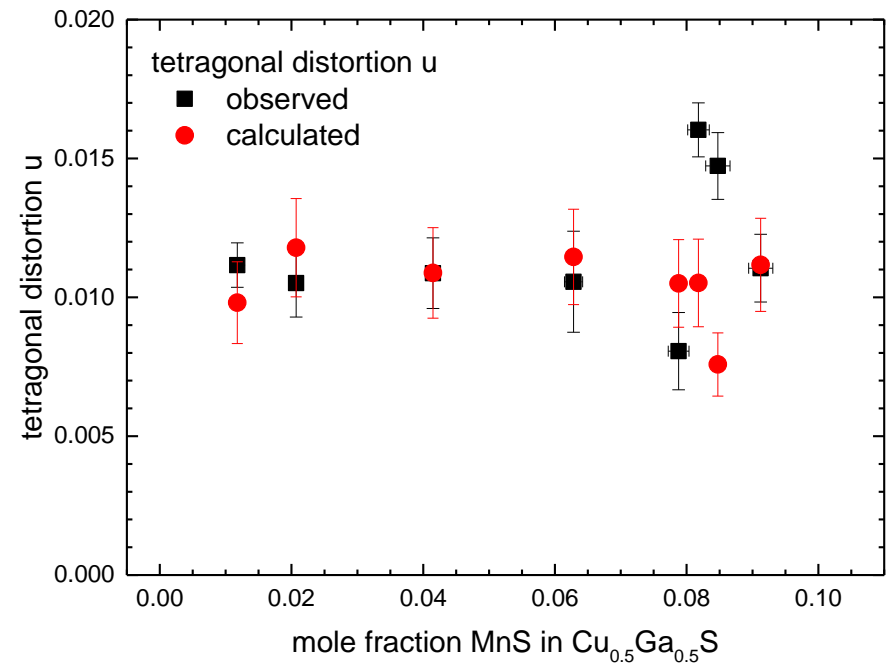

Figure 4 Comparison of experimentally observed and calculated tetragonal distortion $u$ in chalcopyrite-type Mn-substituted $\mathrm{CuGaS}_{2}$. For the calculation the advanced CTB principle formalism was applied. 